\title{
Characterization of $\mathrm{CD8}^{+} \mathrm{T}-$-Cell Responses in the Peripheral Blood and Skin Injection Sites of Melanoma Patients Treated with mRNA Electroporated Autologous Dendritic Cells (TriMixDC-MEL)
}

\author{
Daphné Benteyn, ${ }^{1}$ An M. T. Van Nuffel, ${ }^{1}$ Sofie Wilgenhof, ${ }^{1,2}$ Jurgen Corthals, \\ Carlo Heirman, ${ }^{1}$ Bart Neyns, ${ }^{1,2}$ Kris Thielemans, ${ }^{1,2}$ and Aude Bonehill ${ }^{1}$ \\ ${ }^{1}$ Laboratory of Molecular and Cellular Therapy, Department of Immunology-Physiology and Dendritic Cell Bank, \\ Vrije Universiteit Brussel, 1090 Brussels, Belgium \\ ${ }^{2}$ Department of Medical Oncology, Universitair Ziekenhuis Brussel, 1090 Brussels, Belgium \\ Correspondence should be addressed to Aude Bonehill; aude.bonehill@vub.ac.be
}

Received 4 June 2012; Accepted 21 July 2012

Academic Editor: Julie Curtsinger

Copyright ( 2013 Daphné Benteyn et al. This is an open access article distributed under the Creative Commons Attribution License, which permits unrestricted use, distribution, and reproduction in any medium, provided the original work is properly cited.

\begin{abstract}
Treatment of melanoma patients with mRNA electroporated dendritic cells (TriMixDC-MEL) stimulates T-cell responses against the presented tumor-associated antigens (TAAs). In the current clinical trials, melanoma patients with systemic metastases are treated, requiring priming and/or expansion of preexisting TAA-specific T cells that are able to migrate to both the skin and internal organs. We monitored the presence of TAA-specific $\mathrm{CD} 8^{+} \mathrm{T}$ cells infiltrating the skin at sites of intradermal TriMixDCMEL injection (SKILs) and within the circulation of melanoma patients treated in two clinical trials. In 10 out of fourteen (71\%) patients screened, $\mathrm{CD} 8^{+} \mathrm{T}$ cells recognizing any of the four TAA presented by TriMixDC-MEL cellular vaccine were found in both compartments. In total, 30 TAA-specific T-cell responses were detected among the SKILs and 29 among peripheral blood T cells, of which 24 in common. A detailed characterization of the antigen specificity of CD8 ${ }^{+} \mathrm{T}$-cell populations in four patients indicates that the majority of the epitopes detected were only recognized by $\mathrm{CD} 8^{+} \mathrm{T}$ cells derived from either skin biopsies or peripheral blood, indicating that some compartmentalization occurs after TriMix-DC therapy. To conclude, functional TAA-specific CD $8^{+} \mathrm{T}$ cells distribute both to the skin and peripheral blood of patients after TriMixDC-MEL therapy.
\end{abstract}

\section{Introduction}

Several cancer immunotherapeutic approaches are currently under investigation, amongst which dendritic-cell-based immunotherapy. Dendritic cells (DCs) are potent antigenpresenting cells that can easily be loaded with antigens. Recent improvements of DC therapy include the use of mRNA encoding full-length tumor antigen(s) instead of peptides to load DCs for clinical trials. This results in broader T-cell responses [1-3] and avoids the limitation of known peptides and matching HLA phenotypes.
Monitoring TAA-restricted T-cell responses during treatment is of great importance to investigate the immunogenicity of the vaccine and the potential correlation between the immune response and the clinical outcome of the patients and also for future treatment design. Ideally, immune responses should be monitored within the tumor, but this site is not always accessible. Alternative methods are the characterization of circulating treatment-specific $\mathrm{CD} 8^{+} \mathrm{T}$ cells in the peripheral blood [4-6], or the characterization of treatment-specific skin infiltrating lymphocytes (SKILs) at delayed type hypersensitivity (DTH) sites [7, 8]. Both 
compartments are easily accessible and have advantages and limitations. Immune monitoring of skin biopsies can be performed without prior in vitro T-cell restimulation and highlights the migratory potential of the antigen-specific $\mathrm{CD}^{+} \mathrm{T}$ cells after treatment, but only a limited amount of cells is available. In contrast, peripheral blood screening requires several in vitro restimulations to uncover low frequencies of specific $\mathrm{CD}^{+} \mathrm{T}$ cells; however, enough material is available and pretreatment immune monitoring can be performed without additional invasive intervention. Indeed, all patients undergo a leukapheresis before treatment for the generation of the TriMixDC-MEL vaccine. The remainder of the material is then used for further immune monitoring.

Since, in advanced cancer patients, tumors are located at different anatomical locations, it is of great importance that $\mathrm{T}$ cells have the capacity to migrate to and eradicate tumor cells at different tissue sites. In a mouse study, it has been shown by our group [9] that immunization with TriMix mRNA results in antigen-specific $\mathrm{CD}^{+} \mathrm{T}$ cells located in different organs, including the lymph nodes, spleen, and peripheral blood, highlighting the capacity of the T cells to migrate to different body sites.

With this project, we set out to characterize the immune responses in skin biopsies and peripheral blood of melanoma patients treated with TriMixDC-MEL.

\section{Materials and Methods}

2.1. Patients, Vaccine Preparation, and Treatment Schedule. Fourteen patients with recurrent stage III or stage IV melanoma were recruited in two institutional (UZ Brussels) pilot clinical trials on autologous TriMix-DC treatment (EudraCT2009-015748-40/NCT01066390) [10]. TriMixDCs were manufactured according to a previously described protocol [11]. In brief, immature DCs were coelectroporated with TriMix mRNA (a combination of CD40L, caTLR4, and CD70 encoding mRNA) in combination with one of four mRNAs encoding a TAA (tyrosinase, MAGE-A3, MAGE-C2, or gp100) linked to an HLA class II targeting signal. Genetic constructs encoding these different mRNAs have been described previously [1]. After electroporation, the four different TriMixDC-MEL cellular constituents (i.e., DCs expressing one of the four antigens) were mixed at equal ratios and cryopreserved. Before treatment, an in-process quality control check was performed as well as a quality control check of the final cellular product. The cellular product was thawed 2 to 3 hours before injection. Each patient received 4 DC injections on a biweekly basis, after which immunomonitoring was performed [10]. Patients 72 to 98 (Table 1 ) received 4 times $\pm 43 \times 10^{6}$ DC intradermally (ID), whereas the next four patients (102-116) received a combination of intradermal and intravenous (IV) DCs, whereas the last patient (125) received intravenous DCs only.

\subsection{Monitoring Treatment-Specific T-Cell Responses in Periph-} eral Blood. Immune monitoring of the peripheral blood of the patients was performed as described elsewhere [12]. Pretreatment and posttreatment samples were taken before the first injection and 1 week after the fourth administration, respectively. Briefly, $\mathrm{CD}^{+} \mathrm{T}$ cells were isolated from the nonadherent fraction of the patient's leukapheresis product before and after DC treatment using anti-CD $8^{+}$MACS beads (Miltenyi), obtaining a $>90 \%$ purity (data not shown).

Twenty million $\mathrm{CD} 8^{+} \mathrm{T}$ cells were cocultured with autologous TriMix-DC coelectroporated with one of 4 different tumor antigens at a $10: 1$ ratio in stimulation medium (IMDM, 1\% human $\mathrm{AB}$ serum, $1 \mathrm{mM}$ sodium pyruvate, nonessential amino acids, $0.24 \mathrm{mM}$ L-asparagine, and $0.55 \mathrm{mM}$ L-arginine (all from Lonza, Verviers, Belgium)) without any further addition of exogenous cytokines. CD ${ }^{+}$ $\mathrm{T}$ cells were restimulated weekly. The culture medium was changed when necessary, and after 2 and 3 rounds of stimulation $\mathrm{CD} 8^{+} \mathrm{T}$ cells were harvested and their antigen specificity and function were determined. To this end, they were stimulated overnight with autologous Epstein-Barr Virustransformed B cells (aEBV-B) that were electroporated with the treatment TAAs. Upregulation of CD137, cytokine secretion, and intracellular cytokines were investigated in response to antigen-specific stimulation. To identify the epitopes recognized by the $\mathrm{CD}^{+} \mathrm{T}$ cells, overnight restimulation was performed with aEBV-B cells pulsed with pools of 10 peptides $(10 \mu \mathrm{g} / \mathrm{mL}$ in stimulation medium), or with the individual peptides composing the recognized pools. Peptides were 15 mers, each with 11 amino acids (aa) overlap, and in total spanning the entire TAA sequence. The gp100 protein was spanned by 163 synthetic peptides (16 pools), tyrosinase by 130 peptides (13 pools), MAGE-C2 by 91 peptides ( 9 pools), and MAGE-A3 by 76 peptides ( 8 pools) (all purchased from EMC Microcultures, Tübingen, Germany). Responses were scored based on the percentage CD137 expression and intracellular IFN $\gamma / \mathrm{TNF} \alpha$ (weak $(<10 \%)$, moderate $(10-20 \%)$, or strong $(>20 \%))$. Peptides were considered positive, when the cytokine secretion was increased 2.5-fold compared to a control peptide.

\subsection{Monitoring Treatment-Specific T-Cell Responses in Skin} Biopsies. Immunomonitoring was performed as described elsewhere [8]. Briefly, 1 week after the fourth DC administration, a small amount of TriMixDC-MEL was injected intradermally to induce a delayed type IV hypersensitivity response from which skin biopsies were taken 48 or 72 hours later. After 2.5 weeks of culture in IL-2 (100 IU/mL) supplemented medium, SKILs were harvested and their antigen specificity was determined as for the blood-derived $\mathrm{CD}^{+} \mathrm{T}$ cells.

\section{Results and Discussion}

Immune monitoring of melanoma patients after DC treatment was performed on skin biopsies and on peripheral blood. For 14 patients, we had sufficient material to investigate the presence of antigen-specific $\mathrm{CD}^{+} \mathrm{T}$ cells in both compartments. Cytotoxic $\mathrm{CD}^{+}{ }^{+} \mathrm{T}$-cells are known as key players in tumor immunity. Upon stimulation by recognition of TAA-derived peptides presented by antigen presenting cells, they are capable to kill tumor cells while sparing normal 


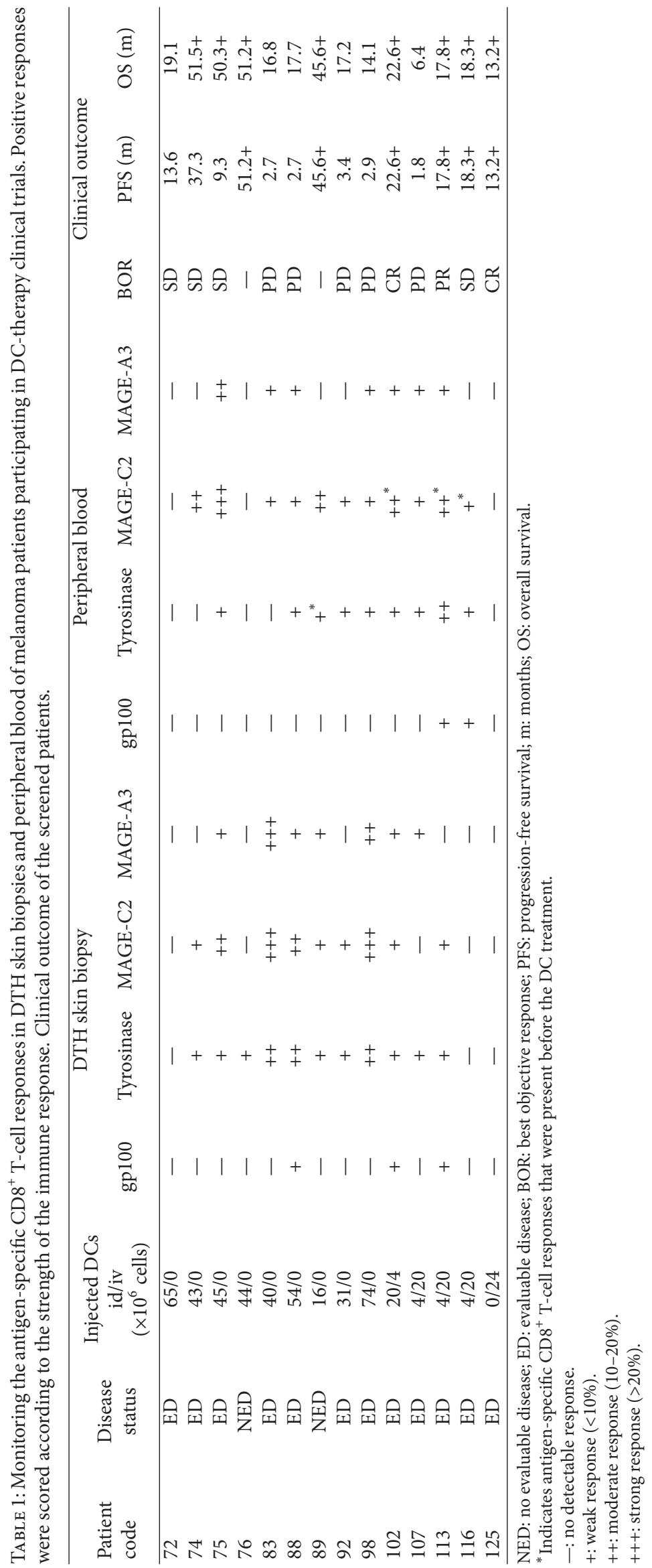


cells, resulting in minimal tissue damage. It is thus important to characterize the $\mathrm{CD}^{+}$T-cell immune response after DC immunotherapy in order to gain knowledge about their capacity to recognize and possibly eradicate tumor cells. An overview of the results is given in Table 1.

Three patients included in this study did not have treatment antigen-specific $\mathrm{T}$ cells in the skin biopsies. Accordingly, in 2 of them, no specific $\mathrm{T}$ cells could be detected in the peripheral blood. In the third patient (116), although the responses were weak, peripheral $\mathrm{CD} 8^{+} \mathrm{T}$ cells recognizing gp100, MAGE-C2, and tyrosinase were found after DC treatment. In contrast, for patient 76, no treatment-specific T cells were found in the blood, whereas a weak tyrosinase-specific response was found in the skin (Table 1).

In the 14 patients tested, we detected 30 positive responses directed against the treatment antigens in the skin biopsies and 29 responses in the peripheral blood, of which 24 in common.

As the patients are treated with an equal ratio mixture of DCs each expressing one out of four different treatment antigens, we also analyzed the responses per antigen. In total, 1/4 gp100-reponders (25\%), 8/12 tyrosinase-responders (67\%), 9/11 MAGE-C2-responders (82\%), and 7/8 MAGE-A3responders $(88 \%)$ had $\mathrm{CD}^{+} \mathrm{T}$ cells that could be detected or found in both compartments.

An advantage of the immune monitoring of the peripheral blood is that preexisting immune responses can be investigated without the need for additional invasive procedures. In this group of patients, we found preexisting responses in 4 out of the 14 patients tested (29\%) (Table 1). In all the patients with a detectable preexisting response, the response was further expanded after TriMixDC-MEL treatment, indicating that our DC therapy is able to expand responses in vivo (Figure 1(a)).

Overall, when looking at the immune responses after DC therapy on the level of the tumor antigen, the $\mathrm{CD} 8^{+}$ T-cell immune responses are very similar in the DTH skin biopsies and the peripheral blood of the patients. This comparison has not been performed by many groups. The group of Gaudernack $[13,14]$ defined a positive DTH immune reaction as induration and/or erythema after intradermal injection of the vaccine but did not investigate the antigenspecificity of the T cells in the DTH skin biopsies. This way, they documented 10/17 [13] and 3/10 [14] positive DTH reactions in their respective studies. On the other hand, antigen-specific $\mathrm{T}$ cells were documented more frequently in peripheral blood after antigen-specific restimulation: 7/17 [13] and 6/10 [14] immune responders were documented in their respective studies. The group of de Vries performed two studies where the antigen-specific $\mathrm{T}$ cells at both sites were investigated, and they detected more treatment antigenspecific T-cell responses in skin biopsies compared to the peripheral blood $[15,16]$. In their first study [15], patients with colorectal cancer were vaccinated with CEA-peptideloaded DCs. Seven out of 10 patients had CEA-specific SKILs detected by direct tetramer analysis, whereas none of the patients had antigen-specific $\mathrm{T}$ cells in the peripheral blood. In a more recent study [16], 27 patients with advanced melanoma were vaccinated ID/IV with a gp100 peptide.
Although the majority of the vaccinated patients induced an induration at the DTH site, only 3 patients had antigenspecific SKILs detected by tetramer staining. No treatment antigen-specific tetramer positive $\mathrm{CD}^{+} \mathrm{T}$ cells could be detected in the blood. This was probably due to a too low frequency of antigen-specific $\mathrm{T}$ cells, since in vitro restimulation indicated that vaccination induced an increase in antigen-specific $\mathrm{CD}^{+}$T cells in the 3 patients with detectable antigen-specific T cells in DTH skin biopsies. Overall, these differences between groups may partly be explained by differences in the type of vaccine, the DC production, maturation, and antigen loading. Indeed, we have treated the patients with TriMixDC-MEL, which have a superior $\mathrm{CD}^{+}$T-cell stimulatory capacity in vitro [17]. Also the route of immunization might play a role [18-20]. In some cases, this difference could be explained by a difference of methods used for monitoring the peripheral blood responses: responses were more frequently observed after in vitro restimulation compared to direct ex vivo analysis, for example, by tetramer staining. Direct tetramer staining is only possible when the patient's HLA type is known and when investigating an immune response directed to a known and defined peptide. With our mRNA-based DC vaccine, the patient's HLA type is not known and the fulllength TAA encoding mRNA ensures presentation of the full antigenic spectrum of epitopes, which impedes the use of direct tetramer staining. On the other hand, high-throughput tetramer-based methods have been developed. These tests could detect a large number of different T-cell epitopes requiring a limited amount of sample volume, without the need for in vitro stimulation [21-23]. Another method that can be used for the detection of antigen-specific responses without in vitro restimulation is ELISpot. However, in our hands, this resulted in a high level of aspecific responses. Unfortunately, the small number of patients investigated in our study does not allow any correlation between antigenspecific responses detected in DTH skin biopsies and peripheral blood and the clinical outcome.

When a positive immune response was observed in the SKILs or the peripheral $\mathrm{CD}^{+} \mathrm{T}$ cells of a patient, we attempted to characterize the antigenic region(s) recognized by the $\mathrm{CD}^{+} \mathrm{T}$ cells. To investigate this, cells were screened for their specificity after stimulation with aEBV-B cells pulsed with pools of 10 overlapping 15-mer peptides. We were able to investigate the antigenic regions recognized by the $\mathrm{CD}^{+} \mathrm{T}$ cells in both compartments of $4 / 10$ patients mounting a $\mathrm{CD}^{+} \mathrm{T}$-cell response in both compartments (Table 2, Figure 1(b)). As this screening method requires high amounts of both skin- and blood-derived $\mathrm{CD}^{+}{ }^{+} \mathrm{T}$ cells, it was not possible to perform this screening for all patients included in this study. SKILs could not always be sufficiently expanded for detection of the fine specificity. Also for peripheral blood screening, leftover material from the vaccinal material was used and available material was a limiting factor in some cases. Nevertheless, in this limited group of patients, both regions containing previously described epitopes as well as regions that do not contain described epitopes were recognized [24]. These broad T-cell responses are a result of the use of full-length mRNA for TriMixDC-MEL treatment, 


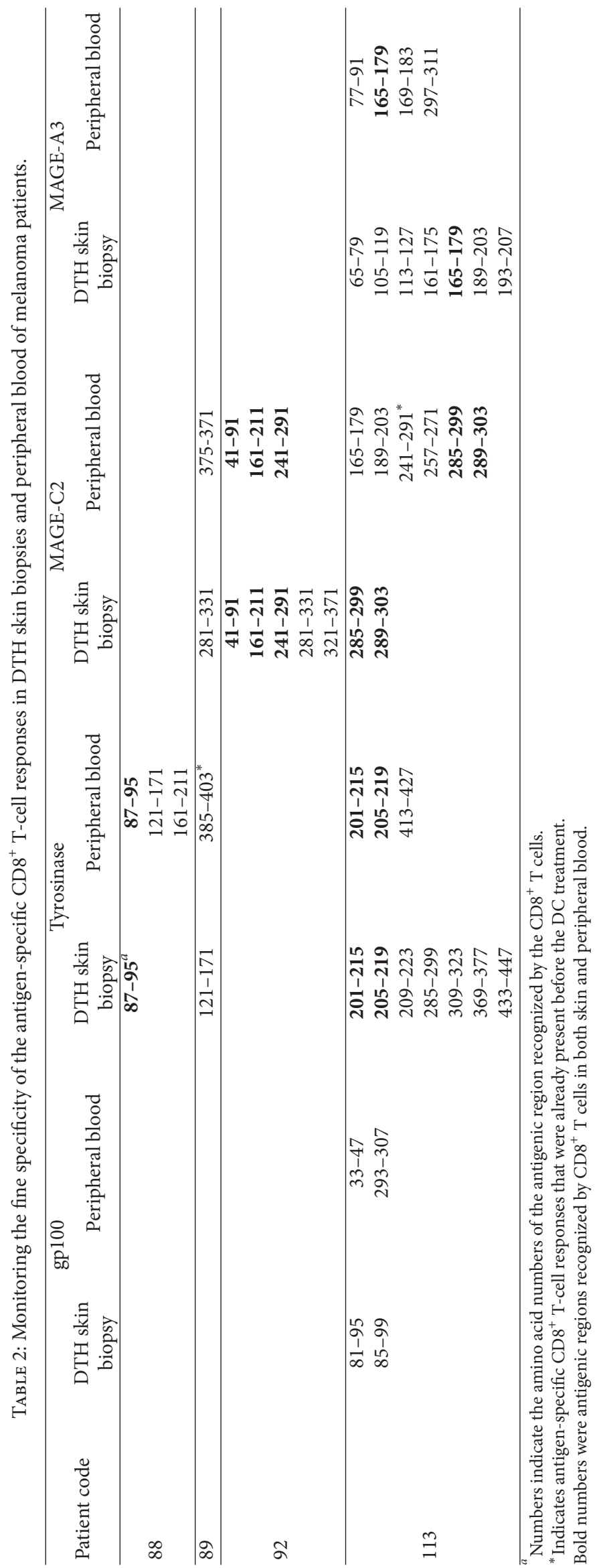




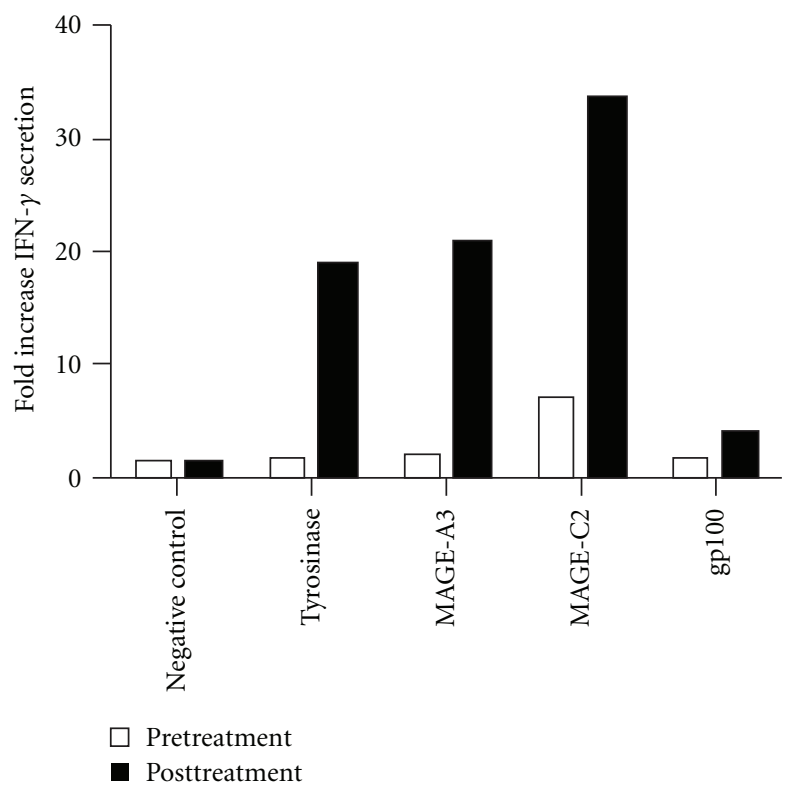

(a) Fold increase of the IFN $\gamma$ secretion of in vitro restimulated $\mathrm{CD} 8^{+}$ $\mathrm{T}$ cells before and after TriMix-DC treatment. T cells were cocultured with aEBV-B cells electroporated with the full-length TAA. mRNA encoding the Nef antigen was used as a control

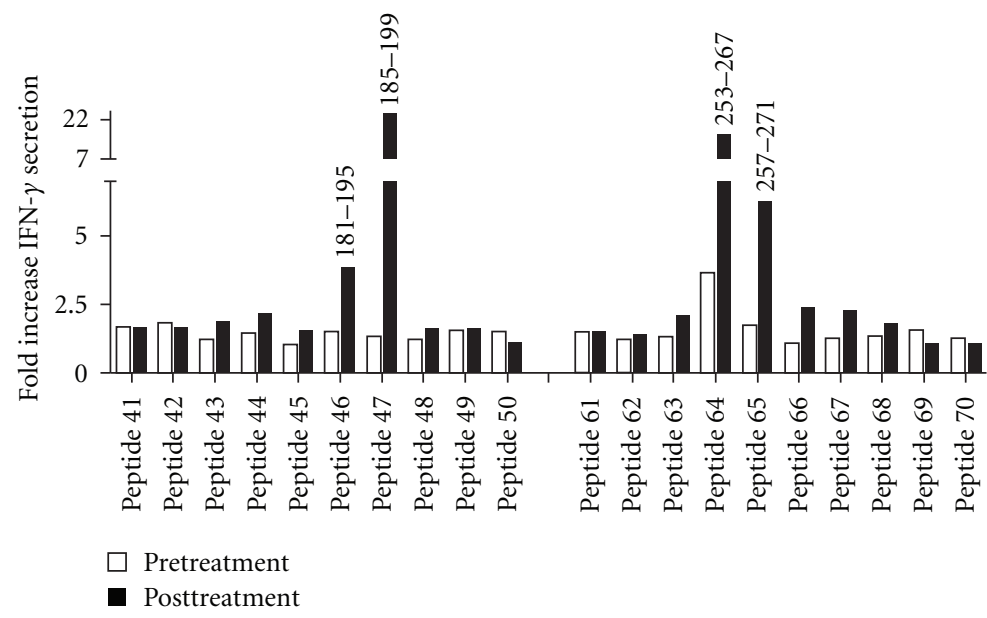

(b) Fold increase in IFN $\gamma$ secretion of $\mathrm{CD} 8^{+} \mathrm{T}$ cells after overnight culture with aEBV$\mathrm{B}$ cells loaded with the individual peptides of pools 5 and 7 of the MAGE-C2 antigen. These pools were found positive after 2 rounds of stimulation. To determine the antigenic region, the cells were screened for their fine specificity in those pools of peptides

FIgURE 1: Treatment antigen-specific $\mathrm{CD}^{+} \mathrm{T}$ cells in peripheral blood.

resulting in the presentation of the complete antigenic repertoire without HLA restriction [1]. Some epitopes were recognized by $\mathrm{CD}^{+} \mathrm{T}$ cells isolated from both compartments, but the majority was either recognized by SKILs or peripheral T cells.

Although only a small number of patients could be screened, these data suggest that there is a partial overlap between the antigenic regions recognized by $\mathrm{CD}^{+} \mathrm{T}$ cells situated in both compartments but that a certain degree of compartmentalization might take place. On the other hand, we cannot exclude that this phenomenon is due to technical limitations of our monitoring techniques. Both DTH skin biopsy and peripheral blood immune monitoring require in vitro culturing of the $\mathrm{T}$ cells and expansion of responding T lymphocytes up to detectable frequencies. As such, a bias towards T cells specific to certain peptides can arise, as some antigen-specific $\mathrm{CD} 8^{+} \mathrm{T}$ cells could be overgrown by others.

In this study, we show that TAA-specific $\mathrm{T}$ cells can be detected in peripheral blood and DTH skin biopsies after TriMixDC-MEL treatment. Our results show that TAAspecific $\mathrm{CD}^{+} \mathrm{T}$ cells circulate in the blood stream. These $\mathrm{T}$ cells have the potential to access every place in the body 
via the circulation. This is of great importance for the eradication of metastasized disease. $T$ cells that were found at the DTH skin biopsy highlight the capacity of the induced TAA-specific $\mathrm{CD}^{+}{ }^{+} \mathrm{T}$ cells to leave the bloodstream and to migrate to tissues with enhanced expression of the treatment TAA. In addition, when tumor lesions are accessible, it should be interesting to also look at this site if antigenspecific $\mathrm{T}$ cells are present. The group of Tjin et al. [5] investigated the antigen specificity of tumor-infiltrating lymphocytes (TILs). They detected more antigen-specific TIL compared to antigen-specific T cells in the peripheral blood, indicating that $\mathrm{T}$-cell responses could be missed when looking only at circulating $\mathrm{T}$ cells. It is thus of great importance to investigate the vaccinal antigen-specific immune response in as many compartments as possible to find all possible antigen-specific T lymphocytes.

\section{General Conclusion and Future Perspectives}

We here report that treatment antigen-specific T cells can be detected both in the skin and the peripheral blood of melanoma patients. Although we see a high overlap between TAA-specific immune responses after TriMixDC-MEL treatment, the observed compartmentalization on the level of recognized antigenic regions highlights the importance of screening for immune responses in different compartments. We hypothesize that the detection of responses against treatment antigens in different body compartments might enable us to refine vaccine strategies and to optimize them.

\section{Acknowledgments}

The authors wish to thank Elsy Vaeremans, Petra Roman, Xavier Debaere, Gwenny De Metter, Abderahim Hbeddou, Inge Betz, Carine Wartel, Chiraz Mahmoud, and Steven Heynderickx for excellent technical assistance and the Department of Radiotherapy of the UZ Brussel for the irradiation of the EBV-B cells. TriMix-DCs are the topic of a current patent application (WO2009/034172). A. Bonehill and K. Thielemans are mentioned as inventors of this application. None of the authors involved in this study receives any form of support or remuneration related to this platform. This work was supported by grants from the Interuniversity Attraction Poles Program-Belgian State-Belgian Science Policy, the National Cancer Plan of the Federal Ministry of Health, the Stichting tegen Kanker, the Vlaamse Liga tegen Kanker, an Integrated Project and a Network of Excellence sponsored by the EU FP-6, an IWT-TBM program, the Fonds voor Wetenschappelijk Onderzoek Vlaanderen (FWO-Vlaanderen), and the Willy Gepts Wetenschappelijk Fonds of the UZ Brussel. S. Wilgenhof is a Ph.D. fellow, and A. Bonehill is a postdoctoral fellow of the FWO-Vlaanderen. D. Benteyn and A. M. T. Van Nuffel contributed equally to this work.

\section{References}

[1] A. M. van Nuffel, D. Benteyn, S. Wilgenhof et al., "Dendritic cells loaded with mRNA encoding full-length tumor antigens prime $\mathrm{CD} 4^{+}$and $\mathrm{CD} 8^{+} \mathrm{T}$ cells in melanoma patients," Molecular Therapy, vol. 20, no. 5, pp. 1063-1074, 2012.

[2] V. F. van Tendeloo, A. van de Veldea, A. van Driesschea et al., "Induction of complete and molecular remissions in acute myeloid leukemia by Wilms' tumor 1 antigen-targeted dendritic cell vaccination," Proceedings of the National Academy of Sciences of the United States of America, vol. 107, no. 31, pp. 13824-13829, 2010.

[3] A. M. van Nuffel, D. Benteyn, S. Wilgenhof et al., "Intravenous and intradermal TriMix-dendritic cell therapy results in a broad T-cell response and durable tumor response in a chemorefractory stage IV-M1c melanoma patient," Cancer Immunology, Immunotherapy, vol. 61, no. 7, pp. 1033-1043, 2012.

[4] A. Bonehill, A. M. T. van Nuffel, J. Corthals et al., "Singlestep antigen loading and activation of dendritic cells by mRNA electroporation for the purpose of therapeutic vaccination in melanoma patients," Clinical Cancer Research, vol. 15, no. 10, pp. 3366-3375, 2009.

[5] E. P. M. Tjin, D. Konijnenberg, G. Krebbers et al., “T-cell immune function in tumor, skin, and peripheral blood of advanced stage melanoma patients: implications for immunotherapy," Clinical Cancer Research, vol. 17, no. 17, pp. 5736-5747, 2011.

[6] E. M. I. Suso, S. Dueland, A. M. Rasmussen et al., "HTERT mRNA dendritic cell vaccination: complete response in a pancreatic cancer patient associated with response against several hTERT epitopes," Cancer Immunology, Immunotherapy, vol. 60, no. 6, pp. 809-818, 2011.

[7] I. J. M. de Vries, M. R. Bernsen, W. J. Lesterhuis et al., "Immunomonitoring tumor-specific $\mathrm{T}$ cells in delayed-type hypersensitivity skin biopsies after dendritic cell vaccination correlates with clinical outcome," Journal of Clinical Oncology, vol. 23, no. 24, pp. 5779-5787, 2005.

[8] A. M. van Nuffel, S. Tuyaerts, D. Benteyn et al., "Epitope and HLA-type independent monitoring of antigen-specific T-cells after treatment with dendritic cells presenting full-length tumor antigens," Journal of Immunological Methods, vol. 377, no. 1-2, pp. 23-36, 2012.

[9] S. van Lint, C. Goyvaerts, S. Maenhout et al., "Preclinical evaluation of TriMix and antigen mRNA-based antitumor therapy," Cancer Research, vol. 72, no. 7, pp. 1661-1671, 2012.

[10] S. Wilgenhof, A. M. T. van Nuffel, J. Corthals et al., "Therapeutic vaccination with an autologous mRNA electroporated dendritic cell vaccine in patients with advanced melanoma," Journal of Immunotherapy, vol. 34, no. 5, pp. 448-456, 2011.

[11] A. M. van Nuffel, J. Corthals, B. Neyns, C. Heirman, K. Thielemans, and A. Bonehill, "Immunotherapy of cancer with dendritic cells loaded with tumor antigens and activated through mRNA electroporation," Methods in Molecular Biology, vol. 629, pp. 405-452, 2010.

[12] A. Bonehill, A. M. T. van Nuffel, J. Corthals et al., "Singlestep antigen loading and activation of dendritic cells by mRNA electroporation for the purpose of therapeutic vaccination in melanoma patients," Clinical Cancer Research, vol. 15, no. 10, pp. 3366-3375, 2009.

[13] P. F. Brunsvig, S. Aamdal, M. K. Gjertsen et al., "Telomerase peptide vaccination: a phase I/II study in patients with nonsmall cell lung cancer," Cancer Immunology, Immunotherapy, vol. 55, no. 12, pp. 1553-1564, 2006. 
[14] R. E. Hunger, K. Kernland Lang, C. J. Markowski et al., "Vaccination of patients with cutaneous melanoma with telomerasespecific peptides," Cancer Immunology, Immunotherapy, vol. 60, no. 11, pp. 1553-1564, 2011.

[15] W. J. Lesterhuis, I. J. M. de Vries, D. H. Schuurhuis et al., "Vaccination of colorectal cancer patients with CEA-loaded dendritic cells: antigen-specific T cell responses in DTH skin tests," Annals of Oncology, vol. 17, no. 6, pp. 974-980, 2006.

[16] W. J. Lesterhuis, G. Schreibelt, N. M. Scharenborg et al., "Wildtype and modified gp100 peptide-pulsed dendritic cell vaccination of advanced melanoma patients can lead to long-term clinical responses independent of the peptide used," Cancer Immunology, Immunotherapy, vol. 60, no. 2, pp. 249-260, 2011.

[17] A. Bonehill, S. Tuyaerts, A. M. T. van Nuffel et al., "Enhancing the t-cell stimulatory capacity of human dendritic cells by coelectroporation with CD40L, CD70 and constitutively active TLR4 encoding mRNA," Molecular Therapy, vol. 16, no. 6, pp. 1170-1180, 2008.

[18] D. W. Mullins, S. L. Sheasley, R. M. Ream, T. N. J. Bullock, Y. X. Fu, and V. H. C.-P. Engelhard, "Route of immunization with peptide-pulsed dendritic cells controls the distribution of memory and effector T cells in lymphoid tissues and determines the pattern of regional tumor control," Journal of Experimental Medicine, vol. 198, no. 7, pp. 1023-1034, 2003.

[19] A. R. Ferguson and V. H. C.-P. Engelhard, "CD8 T cells activated in distinct lymphoid organs differentially express adhesion proteins and coexpress multiple chemokine receptors," Journal of Immunology, vol. 184, no. 8, pp. 4079-4086, 2010.

[20] A. R. Ferguson, L. A. Nichols, A. L. Zarling et al., "Strategies and challenges in eliciting immunity to melanoma," Immunological Reviews, vol. 222, no. 1, pp. 28-42, 2008.

[21] S. R. Hadrup and T. N. Schumacher, "MHC-based detection of antigen-specific $\mathrm{CD}^{+} \mathrm{T}$ cell responses," Cancer Immunology, Immunotherapy, vol. 59, no. 9, pp. 1425-1433, 2010.

[22] R. S. Andersen, P. Kvistborg, T. M. Frøsig et al., "Parallel detection of antigen-specific $\mathrm{T}$ cell responses by combinatorial encoding of MHC multimers," Nature Protocols, vol. 7, pp. 891-902, 2012.

[23] J. H. Velthuis, W. W. Unger, J. R. F. Abreu et al., "Simultaneous detection of circulating autoreactive $\mathrm{CD} 8^{+} \mathrm{T}$-cells specific for different islet cell-associated epitopes using combinatorial MHC multimers," Diabetes, vol. 59, no. 7, pp. 1721-1730, 2010.

[24] P. S. van der Bruggen, N. Vigneron, and B. van den Eynde, "Peptide Database," http://cancerimmunity.org/peptide/. 


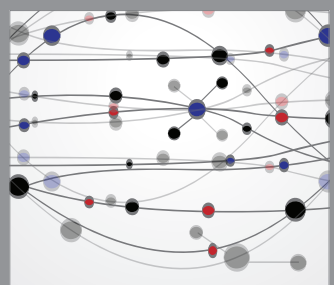

The Scientific World Journal
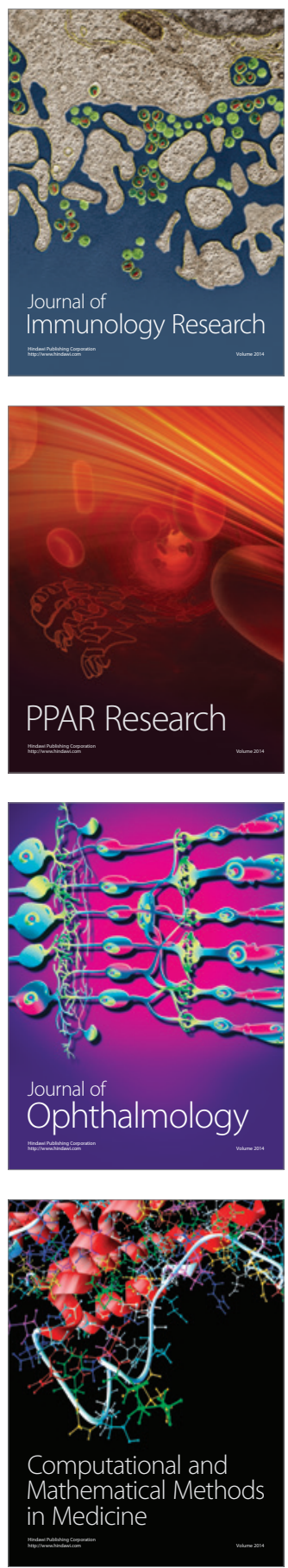

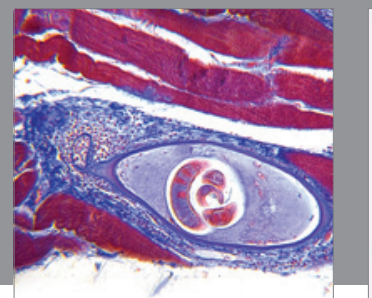

Gastroenterology

Research and Practice
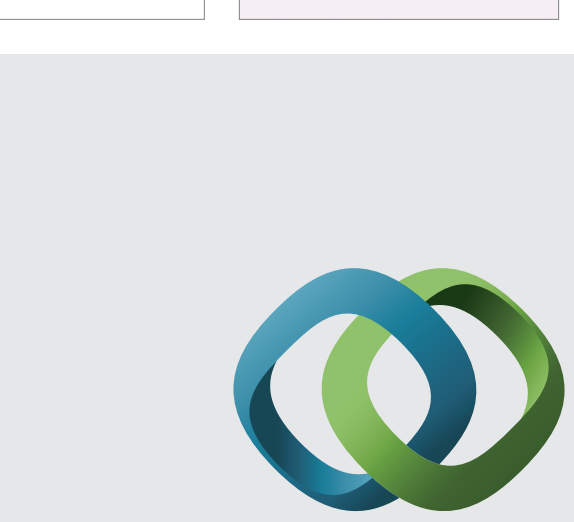

\section{Hindawi}

Submit your manuscripts at

http://www.hindawi.com
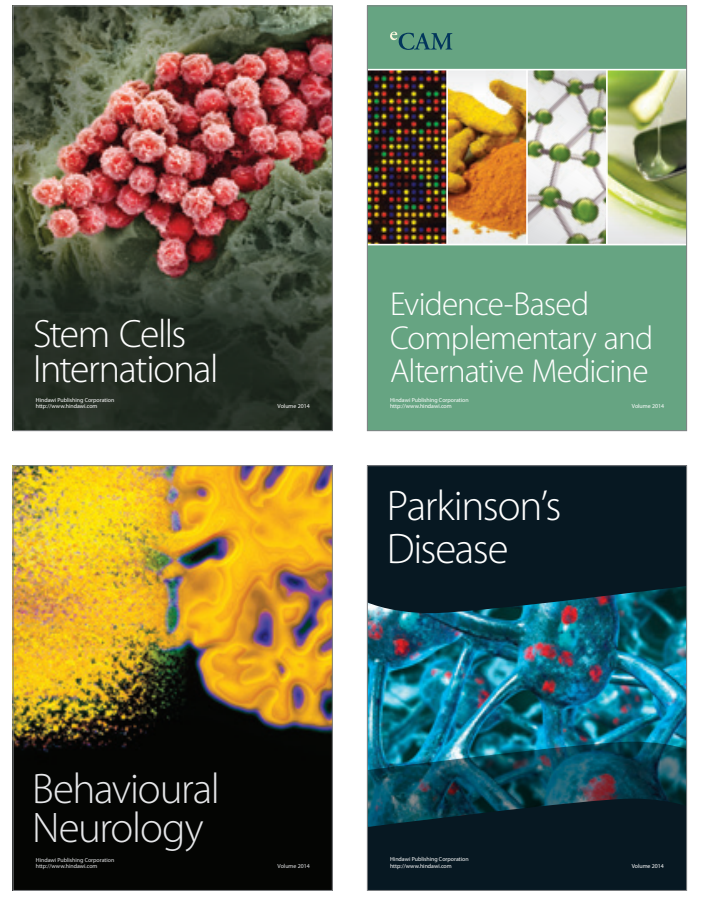
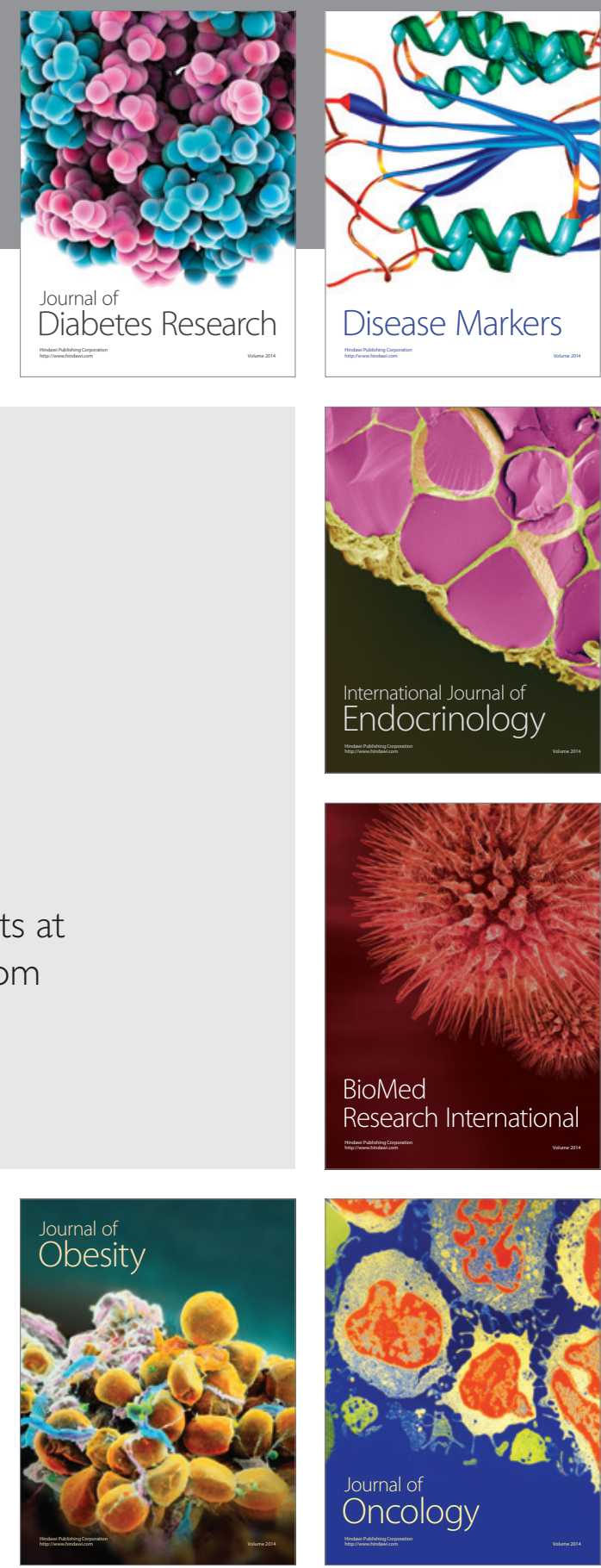

Disease Markers
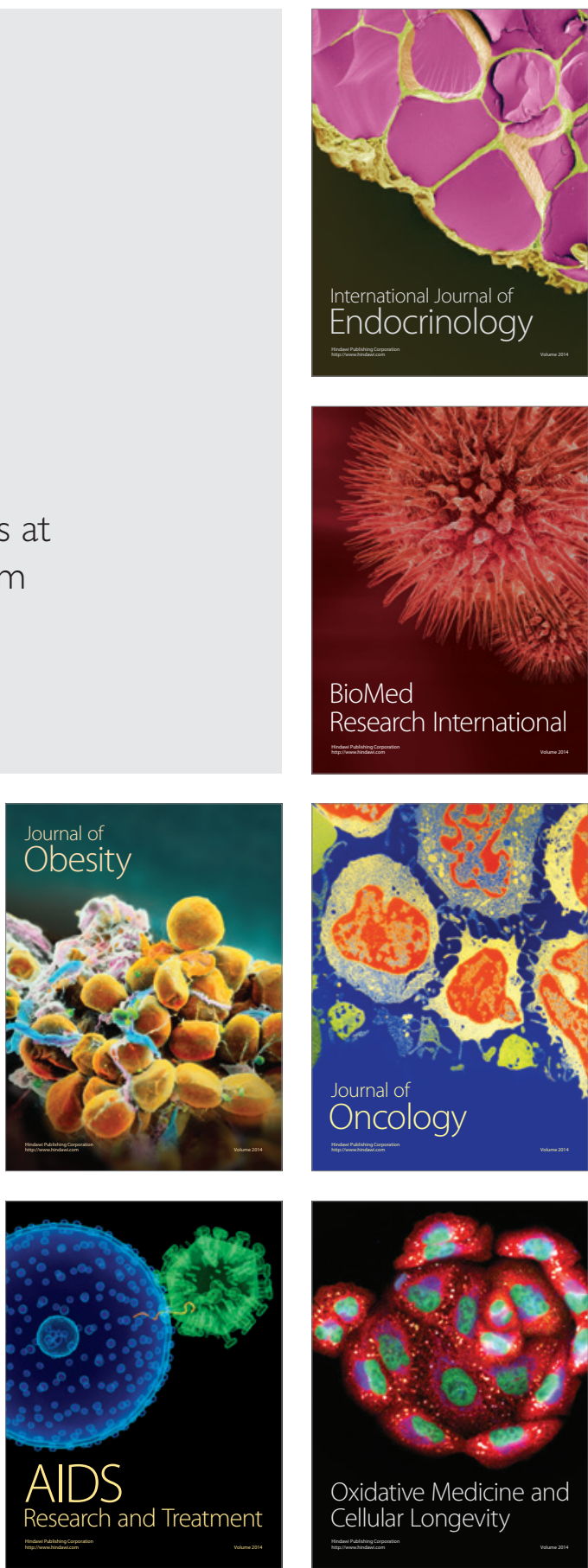